International Journal of Quantum Information

(C) World Scientific Publishing Company

\title{
NON-HERMITIAN DESCRIPTION OF A SUPERCONDUCTING PHASE QUBIT MEASUREMENT
}

\author{
ALEXANDER I. NESTEROV \\ Departamento de Física, CUCEI, Universidad de Guadalajara, Av. Revolución 1500 \\ Guadalajara, Jalisco, 44420, México \\ nesterov@cencar.udg.mx \\ GENNADY P. BERMAN \\ Theoretical Division, Los Alamos National Laboratory \\ Los Alamos, New Mexico 87544, USA \\ gpb@lanl.gov \\ ALAN R. BISHOP \\ The Associate Directorate for Theory, Simulation \& Computation (ADTSC), Los Alamos \\ National Laboratory, Los Alamos, NM 87544, USA \\ arb@lanl.gov \\ Received Day Month Year \\ Revised Day Month Year
}

\begin{abstract}
We present an approach based on a non-Hermitian Hamiltonian to describe the process of measurement by tunneling of a phase qubit state. We derive simple analytical expressions which describe the dynamics of measurement, and compare our results with those experimentally available.
\end{abstract}

Keywords: Superconducting phase qubit, Josephson Junction

\section{Introduction}

The superconducting phase qubit has significant potential applications in quantum information processing due to its convenient functional operations, its capability to reduce the effects of noise and decoherence, and its future utilization in scalable quantum computers 12 . The improvement of the fidelity of the qubit state measurement still remains one of the main issues.

In this paper, we propose an approach based on non-Hermitian Hamiltonians to describe the dynamics of measurement of the superconducting phase qubit state 112134151617. We obtain simple analytical expressions for the probability of tunneling to the continuum. We compare our theoretical results with experiments on superconducting phase qubits $\frac{344}{4}$ and demonstrate that our theoretical predictions are in a good agreement with the available experimental data. 


\section{The non-Hermitian approach to superconducting phase qubit measurement}

Non-Hermitian Hamiltonians naturally appear when the energy spectrum of a quantum system can be formally represented by both quasi-discrete (intrinsic) and continuous parts, and one performs a projection of the total wave function onto the quasi-discrete part of the spectrum $8|9| 10|11| 12 \mid 13$. In this case, the corresponding intrinsic energy levels acquire finite widths which are associated with transitions from the intrinsic states to the continuum. Then, the dynamics of the intrinsic states can be described by the Schrödinger equation with an effective non-Hermitian Hamiltonian 1213 .

We consider the current-biased qubit-continuum detector system based on a Josephson junction, which can be described by the Hamiltonian $(\hbar=1)^{7}$ :

$$
\begin{aligned}
\mathcal{H}_{t}= & \sum_{n} \omega_{n}|n\rangle\left\langle n\left|+\sum_{m \neq n} \beta_{m n}(t)\right| m\right\rangle\left\langle n\left|+\int E\right| E\right\rangle\left\langle E\left|d E+\sum_{n} \int \alpha_{n}(E)\right| E\right\rangle\langle n| d E \\
& +\sum_{n} \int \alpha_{n}^{*}(E)|n\rangle\langle E| d E \quad(m, n=0,1),
\end{aligned}
$$

where $\omega_{n}$ is the energy of level $|n\rangle$. The set of states $\{|n\rangle,|E\rangle\}$ forms a complete Hilbert space basis, so that an arbitrary state vector $|\psi\rangle$ can be expressed as

$$
|\psi\rangle=\sum_{n=0,1} C_{n}|n\rangle+\int C_{E}|E\rangle d E
$$

We use the following notation for qubit states: $|0\rangle=|\downarrow\rangle$ and $|1\rangle=|\uparrow\rangle$. The non-vanishing matrix elements, $\beta_{m n}(t)$, are given by $\beta_{12}=\beta_{21}=\Omega_{0} \cos (\omega t+\varphi)$, where $\Omega_{0}$ is the on-resonance Rabi frequency and $\omega$ is the frequency of the biased ac current, $I_{a c}(t)$. In general, the tunneling amplitudes, $\alpha_{n}(E)$, are complex and depend on the total energy.

Applying the Feshbach projection method $7 / 9|10| 11$, we obtain an effective nonHermitian Hamiltonian, $\tilde{\mathcal{H}}_{\text {eff }}=\mathcal{H}-i \mathcal{W}$, which describes the dynamics of the qubit, where

$$
\mathcal{H}=\left(\begin{array}{cc}
\omega_{1} & \Omega_{0} \cos (\omega t+\varphi) \\
\Omega_{0} \cos (\omega t+\varphi) & \omega_{0}
\end{array}\right)
$$

and

$$
\mathcal{W}=\frac{1}{2}\left(\begin{array}{cc}
\Gamma_{1} & \Gamma_{01} \\
\Gamma_{10} & \Gamma_{0}
\end{array}\right)
$$

Here we consider the case in which the $\alpha_{n}$ 's are real constants. Then, in terms of tunneling amplitudes, the rates of tunneling into the continuum are: $\Gamma_{0}=2 \pi \alpha_{0}^{2}$, $\Gamma_{1}=2 \pi \alpha_{1}^{2}$ and $\Gamma_{10}=\Gamma_{01}=\sqrt{\Gamma_{0} \Gamma_{1}}$.

The state of the qubit is controlled by a current $I(t)=I_{d c}-I_{\mu w} \cos (\omega t+\varphi)$ with a dc bias current, $I_{d c}$, and a time-varying bias current, $I_{a c}=I_{\mu w} \cos (\omega t+\varphi)$, with frequency, $\omega\left[14\right.$. The bias current, $I_{d c}$, can be chosen so that the tunneling 
is mostly from the upper level $|1\rangle$. (See Fig. 11) In what follows, we study the dynamics of quantum measurement by tunneling of a superconducting phase qubit in the limit $\Gamma_{1} \gg \Gamma_{0}, \gamma_{10}, \gamma_{\varphi}$, where $\gamma_{\varphi}$ is the dephasing rate. (We will discuss interaction with environment elsewhere a.)

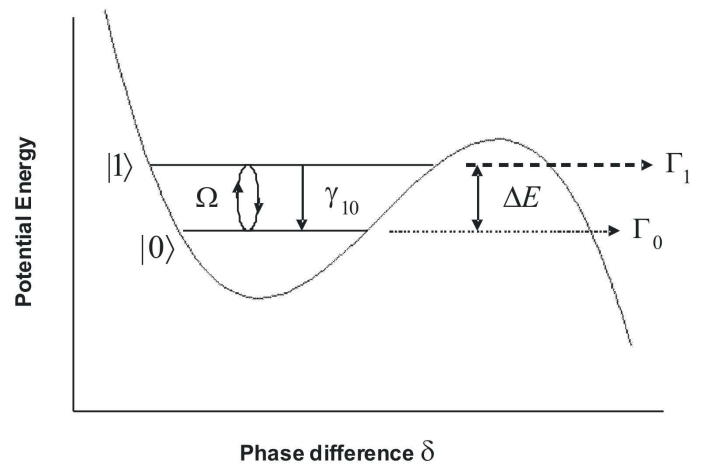

Fig. 1. A superconducting phase qubit radiated by a microwave field; $\delta$ is the gauge-invariant phase difference of superconducting order parameter across the junction; $\gamma_{10}$ denotes the rate of energy relaxation from $|1\rangle$ to $|0\rangle$ and $\Gamma_{1}\left(\Gamma_{0}\right)$ denotes the tunneling rate from level $|1\rangle(|0\rangle)$.

\subsection{Rotating wave approximation}

In the rotating wave approximation, by removing fast oscillations, we find that $\tilde{\mathcal{H}}_{\text {eff }} \rightarrow \tilde{\mathcal{H}}$, where

$$
\tilde{\mathcal{H}}=\frac{1}{2}\left(\begin{array}{cc}
\lambda_{0}+\Delta-i \Gamma_{1} & \Omega_{0} e^{-i \varphi} \\
\Omega_{0} e^{i \varphi} & \lambda_{0}-\Delta-i \Gamma_{0}
\end{array}\right)
$$

Here $\lambda_{0}=\omega_{0}+\omega_{1} ; \Delta=\omega_{10}-\omega$ is the detuning (with $\omega$ being the microwave frequency; and we denote $\left.\omega_{10}=\omega_{1}-\omega_{0}\right)$. We neglect here the off-diagonal term $\Gamma_{01}$, which describe the interaction between the two tunneling channels. We let the wave function $|u(t)\rangle=C_{1}(t)|1\rangle+C_{0}(t)|0\rangle$ describe the qubit state. We assume below that $\langle u(0) \mid u(0)\rangle=1$. Then the solution of the Schrödinger equation with the


master equation in the Lindblad form, $\dot{\rho}=-i[\mathcal{H}, \rho]+L \rho-\{\mathcal{W}, \rho\}$, where $\{\mathcal{W}, \rho\}=\mathcal{W} \rho+\rho \mathcal{W}$ and $L \rho=\frac{1}{2} \sum_{k=1}^{2}\left\{2 L_{k} \rho L_{k}^{\dagger}-L_{k}^{\dagger} L_{k} \rho-\rho L_{k}^{\dagger} L_{k}\right\}$, and transition operators, $L_{k}$, describe the coupling to the environment 1516 . 
non-Hermtian Hamiltonian (5) can be written as

$$
\begin{aligned}
& C_{1}(t)=e^{-i \tilde{\lambda}_{0} t / 2}\left(\left(\cos \frac{\Omega t}{2}-i \cos \theta \sin \frac{\Omega t}{2}\right) C_{1}(0)-i e^{-i \varphi} \sin \theta \sin \frac{\Omega t}{2} C_{0}(0)\right), \\
& C_{0}(t)=e^{-i \tilde{\lambda}_{0} t / 2}\left(\left(\cos \frac{\Omega t}{2}+i \cos \theta \sin \frac{\Omega t}{2}\right) C_{0}(0)-i e^{i \varphi} \sin \theta \sin \frac{\Omega t}{2} C_{1}(0)\right), \\
& \rho_{11}(t)=e^{-\Gamma t}\left|\left(\cos \frac{\Omega t}{2}-i \cos \theta \sin \frac{\Omega t}{2}\right) C_{1}(0)-i e^{-i \varphi} \sin \theta \sin \frac{\Omega t}{2} C_{0}(0)\right|^{2}, \\
& \rho_{00}(t)=e^{-\Gamma t}\left|\left(\cos \frac{\Omega t}{2}+i \cos \theta \sin \frac{\Omega t}{2}\right) C_{0}(0)-i e^{i \varphi} \sin \theta \sin \frac{\Omega t}{2} C_{1}(0)\right|^{2},
\end{aligned}
$$

where $\tilde{\lambda}_{0}=\omega_{0}+\omega_{1}-i \Gamma, \Gamma=\left(\Gamma_{0}+\Gamma_{1}\right) / 2, \cos \theta=\left(\Delta-i\left(\Gamma-\Gamma_{0}\right)\right) / \Omega$ and $\sin \theta=$ $\Omega_{0} / \Omega$, the complex Rabi frequency is $\Omega=\sqrt{\Omega_{0}^{2}+\left(\Delta-i\left(\Gamma-\Gamma_{0}\right)\right)^{2}}$; the upper level population is $\rho_{11}(t)=\left|C_{1}(t)\right|^{2}$ and the lower level population is $\rho_{00}=\left|C_{0}(t)\right|^{2}$.

In what follows we compare our theoretical results with experiments on quantum

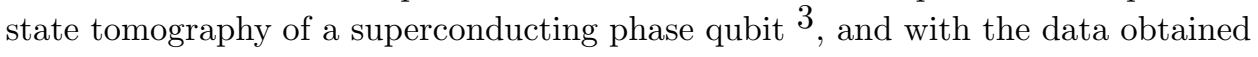
in the experiments observing coherent temporal oscillations between the quantum states of a Josephson tunnel junction 4 . In both experiments the tunneling rate from level $|1\rangle$ is more than $10^{3}$ times that from level $|0\rangle$. So the total tunneling rate to the continuum is determined by the population of the upper level. The bias current was chosen so that the tunneling from level $|0\rangle$ is "frozen out" and escapes mostly from the upper level $3 \sqrt{4}$. Therefore, the time-dependent tunneling probability is defined by the upper level population $\rho_{11}(t)$.

In Fig. 2 we present our analytical solution for evolution of the Bloch vector, $\mathbf{n}(t)=\langle u(t)|\boldsymbol{\sigma}| u(t)\rangle$. The decay rate, $\Gamma$, and the on-resonance Rabi frequency, $\Omega_{0}$, are taken from 3 : $\Gamma=0.035 \mathrm{~ns}^{-1}, \Omega_{0} /(2 \pi)=80 \mathrm{MHz}$. Note, that the following relation between the density matrix and the Bloch vector, $\rho=(n \mathbb{1}+\mathbf{n} \cdot \boldsymbol{\sigma}) / 2$, takes place. Our theoretical predictions shown in Fig. 2 are in a good agreement with the experimental data and with the numerical calculations reported in 3 .

In Fig. 3 we compare our theoretical results with the reported experimental data 4 on evidence for the Rabi oscillations in a current-biased qubit based on the Josephson junction. The Rabi frequency, $|\Omega|$, and decay parameter, $\Gamma$, are chosen as in 4. $|\Omega|=8.9 \cdot 10^{6} \mathrm{rad} / \mathrm{s}$ and $\Gamma=0.204 \mu \mathrm{s}^{-1}$. The initial state $|u(0)\rangle=0.956|0\rangle+$ $0.291|1\rangle$, the detuning $\Delta$, and the on-resonance Rabi frequency, $\Omega_{0}$, are obtained by the fitting data to Eq. (8). We see good agreement between the experimental data and the predicted theoretical behavior. We find that our time-dependent upperlevel population is a better fit to the experimental data than the theoretical results presented in Ref. ${ }^{4}$ by Eq.(5). However, additional information on the experimental data is required for better comparison.

In the experiment reported in 4 , the total tunneling probability was identified as the upper level population given by,

$$
\rho_{11}(t)=e^{-\Gamma t} \frac{\Omega_{0}^{2}}{|\Omega|^{2}}\left|\sin \frac{\Omega t}{2}\right|^{2} .
$$



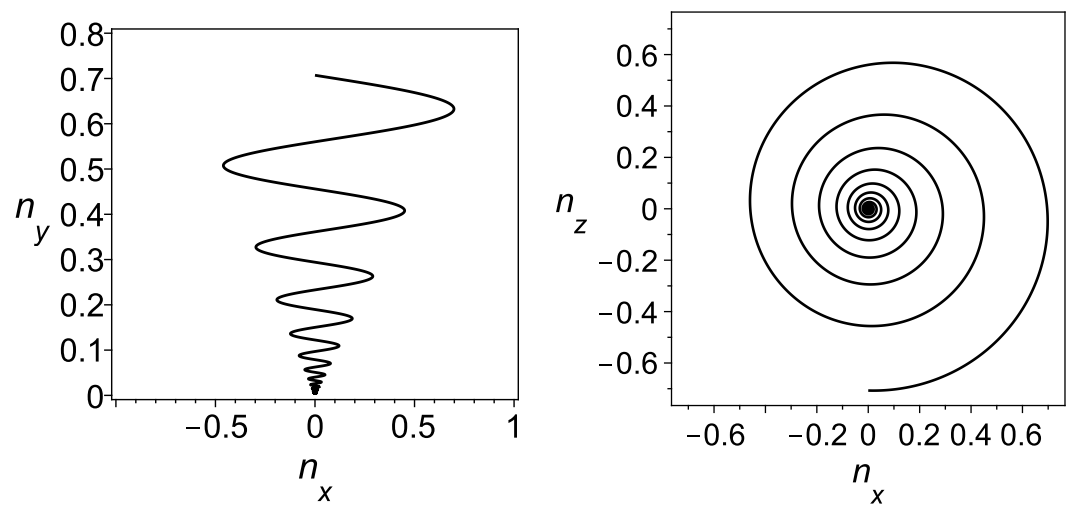

Fig. 2. Projections of the Bloch vector on the $x y$ (left) and $x z$ (right) planes of the Bloch sphere. The parameters are $\Delta=0, \varphi=-\pi / 2$ and the initial state is: $\mathbf{n}(0)=\frac{1}{\sqrt{2}}(0,1,-1)$.

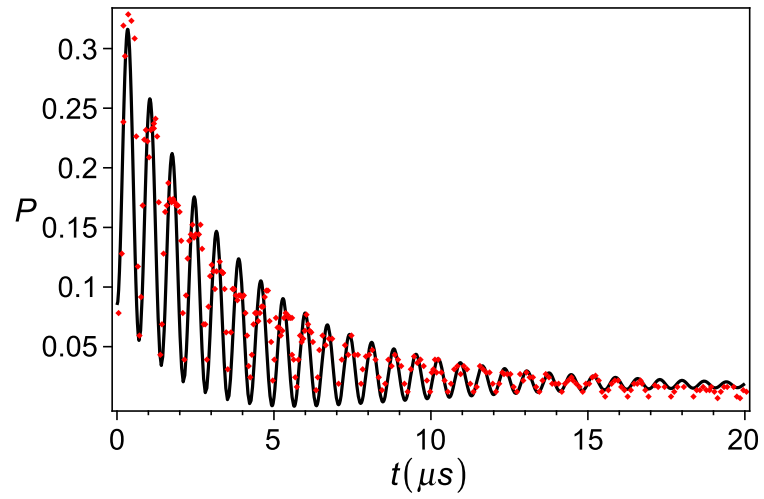

Fig. 3. The probability, $P(t)$, of tunneling from the upper level to the continuum as a function of time. $\left(\Omega_{0} /(2 \pi)=0.47 \mathrm{MHz}, \Delta /(2 \pi)=1.34 \mathrm{MHz}, \Gamma=0.204 \mu \mathrm{s}^{-1}, \Gamma_{0}=0.4 \cdot 10^{-3} \mu s^{-1}\right.$. The diamonds (red) are the data and the black solid line is the best fit to the oscillatory part of Eq. (8).

This is a particular case of our expression (8), corresponding to the choice $C_{1}(0)=0$.

\subsection{Tunneling into the continuum at zero ac current}

The fast frequency detector described in 17 is based on a superconducting phase qubit tunneling into continuum. In particular, the measurement is performed with ac current, $I_{\mu w}$, equal to zero. Suppose, that initially the superconducting phase qubit is prepared as a superposition of ground and excited states. Writing $|u(t)\rangle=$ $C_{1}(t)|1\rangle+C_{0}(t)|0\rangle$, we obtain the time-dependent amplitudes $C_{0}(t)$ and $C_{1}(t)$ as 
solution of the Schrödinger equation

$$
\begin{aligned}
& C_{1}(t)=e^{-i \tilde{\lambda}_{0} t / 2}\left(\left(\cos \frac{\Omega t}{2}-\frac{\Gamma-\Gamma_{0}+i \omega_{10}}{\Omega} \sin \frac{\Omega t}{2}\right) C_{1}(0)-\frac{\Gamma_{01}}{\Omega} \sin \frac{\Omega t}{2} C_{0}(0)\right), \\
& C_{0}(t)=e^{-i \tilde{\lambda}_{0} t / 2}\left(\left(\cos \frac{\Omega t}{2}+\frac{\Gamma-\Gamma_{0}+i \omega_{10}}{\Omega} \sin \frac{\Omega t}{2}\right) C_{0}(0)-\frac{\Gamma_{01}}{\Omega} \sin \frac{\Omega t}{2} C_{1}(0)\right),
\end{aligned}
$$

where $\Omega=\sqrt{\omega_{10}^{2}-2 i \omega_{10}\left(\Gamma-\Gamma_{0}\right)-\Gamma^{2}}$ is the complex Rabi frequency. In the limit $\omega_{10} \gg \Gamma_{1}$, we have

$$
|u(t)\rangle=e^{-i \omega_{0} t} e^{-\Gamma_{0} t / 2} C_{0}(0)|0\rangle+e^{-i \omega_{1} t} e^{-\Gamma_{1} t / 2} C_{1}(0)|1\rangle .
$$

This simple formula is consistent with the known experimental data and the theoretical description of a partial-collapse measurement 6/18/19.

The probability of escape from either $|1\rangle$ or $|0\rangle$ is given by

$$
P_{e s c}(t)=1-\rho_{11}(t)-\rho_{00}(t),
$$

and in the same limit as above, $\omega_{10} \gg \Gamma_{1}$, we obtain

$$
P_{e s c}(t)=1-\rho_{11}(0) e^{-\Gamma_{1} t}-\left(1-\rho_{11}(0)\right) e^{-\Gamma_{0} t} .
$$

As one can see, the tunneling into the continuum is described by a doubleexponential decay function $P_{e s c}$. This behavior of the probability of escape was observed in the experiment on measurement of dissipation-induced decoherence in a Josephson junction 20 .

While the contribution of the off-diagonal term, $\Gamma_{01}$, in the experiments discussed above is negligible, it can be more significant in the fast readout measurement of the phase qubit with a duration of measurement of approximately $\sim 3 \mathrm{~ns}$ 21. To estimate the contribution of the non-diagonal term, $\Gamma_{01}$, we introduce the
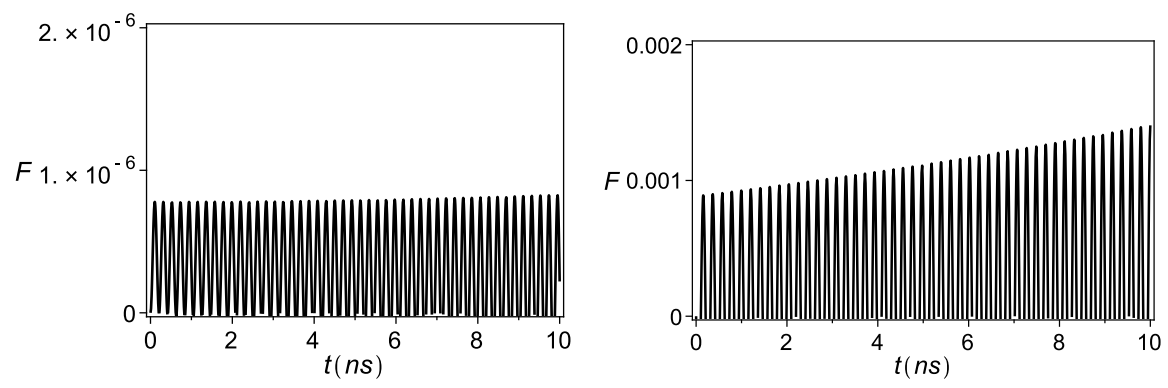

Fig. 4. Deviation of population, $F(t)$, as function of time. Left: $C_{0}(0)=0, C_{1}(0)=1$. Right: $C_{0}(0)=C_{1}(0)=1 / \sqrt{2}$.

function, $F(t)$, which characterizes the difference in probabilities when $\Gamma_{01} \neq 0$ and 
$\Gamma_{01}=0:$

$$
F(t)=\frac{\rho_{11}\left(\Gamma_{01}, t\right)-\rho_{11}(0, t)}{\rho_{11}(0, t)} .
$$

In Fig. 4 we present the results of computation for fast readout with the parameters being chosen as in 21: $\Gamma_{1}=0.1 \mathrm{GHz}, \omega_{10} / 2 \pi=5 \mathrm{GHz}$ and $\Gamma_{1} / \Gamma_{0}=150$. One can see that, depending on the initial conditions, the contribution from the interaction between the two tunneling channels can be up to $\approx 0.1 \%$.

\section{Concluding remarks}

In conclusion, we have presented a non-Hermitian description of the dynamics of measurement of a supeconducting phase qubit. We have shown that our theoretical predictions are in a good agreement with the experimental data obtained in various experiments on superconducting phase qubits. We believe that the approach based on non-Hermitian Hamiltonians can simplify the theoretical description of the dynamics of the phase qubit measurement by tunneling to the continuum (in many cases and especially for a multi-qubit register).

This work was carried out under the auspices of the National Nuclear Security Administration of the U.S. Department of Energy at Los Alamos National Laboratory under Contract No. DE-AC52-06NA25396. The work by AIN was supported by research grant SEP-PROMEP 103.5/04/1911. The work by GPB was partly supported by the IARPA.

\section{References}

1. John M. Martinis, S. Nam, J. Aumentado, and C. Urbina. Rabi Oscillations in a Large Josephson-Junction Qubit. Phys. Rev. Lett., 89(11):117901, Aug 2002.

2. John M. Martinis. Superconducting phase qubits. Quantum Information Processing, 8(2):81 - 103, June 2009.

3. Matthias Steffen, M. Ansmann, R. McDermott, N. Katz, Radoslaw C. Bialczak, Erik Lucero, Matthew Neeley, E. M. Weig, A. N. Cleland, and John M. Martinis. State Tomography of Capacitively Shunted Phase Qubits with High Fidelity. Phys. Rev. Lett., 97(5):050502, 2006.

4. Yang Yu, Siyuan Han, Xi Chu, Shih-I Chu, and Zhen Wang. Coherent temporal oscillations of macroscopic quantum states in a Josephson junction. Science, 296(5569):889$892,2002$.

5. Matthias Steffen, John M. Martinis, and Isaac L. Chuang. Accurate control of Josephson phase qubits. Phys. Rev. B, 68(22):224518, Apr 2003.

6. Nadav Katz, Matthew Neeley, M. Ansmann, Radoslaw C. Bialczak, M. Hofheinz, Erik Lucero, A. O'Connell, H. Wang, A. N. Cleland, John M. Martinis, and Alexander N. Korotkov. Reversal of the Weak Measurement of a Quantum State in a Superconducting Phase Qubit. Phys. Rev. Lett., 101(20):200401, 2008.

7. Alexander I. Nesterov and Gennady P. Berman. Non-Hermitian description of superconducting phase qubits. In preparation, 2009.

8. U. Fano. Effects of configuration interaction on intensities and phase shifts. Phys. Rev., 124(6):1866-1878, Dec 1961. 
9. I. Rotter. Dynamics of quantum systems. Phys. Rev. E, 64(3):036213, 2001.

10. Ingrid Rotter. A Non-Hermitian Hamilton operator and the physics of open quantum systems. J. Phys. A, 42(15):153001, 2009.

11. Alexander Volya and Vladimir Zelevinsky. Discrete and continuum spectra in the unified shell model approach. Phys. Rev. Lett., 94(5):052501, 2005.

12. P. L. Knight, M. A. Lauder, and B. J. Dalton. Laser-induced continuum structure. Physics Reports, 190(1):1 - 61, 1990.

13. E. Kyrola. N level and continuum. J. Phys. B, 19:1437 - 1448, 1986.

14. John M. Martinis, S. Nam, J. Aumentado, K. M. Lang, and C. Urbina. Decoherence of a superconducting qubit due to bias noise. Phys. Rev. B, 67(9):094510, Mar 2003.

15. G. Lindblad. On the generators of quantum dynamical semigroups. Commun. Math. Phys., 48:119, 1976.

16. H. Carmichael. An Open Systems Approach to Quantum Optics. Springer, N. Y., 1993.

17. Alexander N. Korotkov. Quantum efficiency of binary-outcome detectors of solid-state qubits. Phys. Rev. B, 78(17), 2008.

18. N. Katz, M. Ansmann, Radoslaw C. Bialczak, Erik Lucero, R. McDermott, Matthew Neeley, Matthias Steffen, E. M. Weig, A. N. Cleland, John M. Martinis, and A. N. Korotkov. Coherent State Evolution in a Superconducting Qubit from Partial-Collapse Measurement. Science, 312(5779):1498-1500, 2006.

19. Leonid P. Pryadko and Alexander N. Korotkov. Coherence of a josephson phase qubit under partial-collapse measurement. Phys. Rev. B, 76(10), 2007.

20. Siyuan Han, Yang Yu, Xi Chu, Shih-I Chu, and Zhen Wang. Time-Resolved Measurement of Dissipation-Induced Decoherence in a Josephson Junction. Science, 293(5534):1457-1459, 2001.

21. K. B. Cooper, Matthias Steffen, R. McDermott, R. W. Simmonds, Seongshik Oh, D. A. Hite, D. P. Pappas, and John M. Martinis. Observation of Quantum Oscillations between a Josephson Phase Qubit and a Microscopic Resonator Using Fast Readout. Phys. Rev. Lett., 93(18):180401, 2004. 